\title{
AVALIAÇÃO DA CONCENTRAÇÃO DE ALGUNS ÍONS METÁLICOS EM DIFERENTES ESPÉCIES DE LÍQUENS DO CERRADO SUL-MATO-GROSSENSE
}

\author{
Jorge Luiz Raposo Junior*, Nilva Ré-Poppi e Neli Kika Honda \\ Departamento de Química, Universidade Federal do Mato Grosso do Sul, CP 549, 79070-900 Campo Grande - MS, Brasil
}

Recebido em 17/2/06; aceito em 4/8/06; publicado na web em 19/1/07

\begin{abstract}
EVALUATION OF CONCENTRATION OF SOME METAL IONS IN DIFFERENT LICHEN SPECIES OF THE SUL-MATOGROSSENSE CERRADO. Concentrations of $\mathrm{Fe}, \mathrm{Mn}, \mathrm{Co}, \mathrm{Cr}, \mathrm{Zn}$ and $\mathrm{Cu}$ were determinated using flame atomic absorption spectrometry in nine lichen species of the Sul-Mato-Grossense cerrado. The average metal ion concentrations varied in the following ranges: $\mathrm{Fe}, 248.41-1568.01 ; \mathrm{Mn}, 98.50-397.33$; $\mathrm{Co}, 10.08-24.81$; $\mathrm{Cr}, 18.24-44.26 ; \mathrm{Zn}, 14.62-34.79$ and $\mathrm{Cu}, 3.23-7.57 \mathrm{mg} \mathrm{kg}^{-1}$. Statistical analysis (Pearson and Cluster) applied to the metal ion concentrations indicated that the accumulation of these ions can be due to several anthropogenic sources including agricultural activities, mineral exploration, biomass burning, soil mineral composition and leather tanning processes by chromium.
\end{abstract}

Keywords: lichens; heavy metals; multivariate statistics.

\section{INTRODUÇÃO}

Líquens são estruturas resultantes da simbiose entre um fungo e uma ou mais algas ou cianobactérias ${ }^{1}$. São encontrados nos mais variados ambientes desde o nível do mar até as montanhas mais altas, em desertos onde a temperatura é bastante variável e em regiões polares, com temperaturas extremamente baixas, porém, são relativamente raros em matas com baixa luminosidade ${ }^{2}$. Desenvolvem-se sobre os mais variados substratos, tais como, córtex de árvores, rochas ácidas ou alcalinas, folhas e outros. O desenvolvimento dos líquens sobre um dado substrato pode depender das características físicas, como rugosidade, porosidade, dureza, exposição à luz, pH, presença de partículas no ar, umidade, ventos e temperatura ${ }^{3}$.

Os líquens dependem da nutrição mineral para seu crescimento e metabolismo, fornecida pela deposição de elementos por vias seca e úmida na forma de sais solúveis e partículas. Os espaços intercelulares do talo do líquen podem acumular e reter espécies químicas adsorvidas a partículas ou ligadas a sítios de troca iônica. Muitos elementos são acumulados em concentrações que excedem as necessidades metabólicas do líquen ${ }^{4}$. A maior parte das espécies de líquens apresenta crescimento lento e mantém uniformidade morfológica durante o ciclo de vida; sendo que muitas espécies têm ampla distribuição geográfica. Devido a isso os líquens são úteis como biomonitores em estudos de deposição atmosférica ${ }^{5}$. O biomonitoramento pode ser definido como um método experimental indireto de se verificar a existência de poluentes em uma dada região, utilizando-se de organismos vivos que respondem ao estresse causado pela poluição, apresentando modificações nos ciclos vitais ou acumulando os elementos poluentes ${ }^{6-8}$.

Estudos que avaliam a acumulação de elementos aerotransportados direcionados a líquens a partir de uma fonte de poluição específica também são comuns ${ }^{9-12}$. Há também estudos focalizados na retenção, localização, liberação, tolerância e toxicidade de diferentes elementos ${ }^{13-16}$.

A morfologia do líquen influencia na taxa de acumulação dos elementos provenientes da atmosfera ${ }^{17}$. Os líquens aqui estudados são de talos foliosos e fruticosos, que são bem distintos quanto à forma de crescimento. Líquens foliosos são dorsiventrais e possuem talos com superfície bem definida e são presos ao substrato em toda a extensão. Os líquens fruticosos são densamente ramificados e com um único ponto de fixação ${ }^{4}$. A forma de crescimento ordena a orientação na superfície do líquen para acumulação do elemento aerotransportado; alguns estudos têm mostrado que os líquens foliosos acumulam quantidades maiores de elementos aerotransportados quando comparados com líquens fruticosos ${ }^{18}$.

O presente estudo visa a determinação dos íons metálicos Zn, $\mathrm{Fe}, \mathrm{Cr}, \mathrm{Co}, \mathrm{Cu}$ e Mn pela espectrometria de absorção atômica de chama em líquens do cerrado sul-mato-grossense coletados em uma região próxima à cidade de Aquidauana, para verificar os níveis de contaminação ambiental e correlacioná-los com as atividades antropogênicas mais freqüentes na região.

\section{PARTE EXPERIMENTAL}

\section{Localização e climatologia do local de coleta}

Os vilarejos de Palmeiras e Piraputanga, a 109 km da capital Campo Grande, pertencem ao município de Aquidauana/MS, cuja área da unidade territorial é de $16.958,50 \mathrm{~km}^{2}$ a $181 \mathrm{~m}$ de altitude. Estes vilarejos estão localizados a $20^{\circ} 27^{\prime} \mathrm{S}$ e $55^{\circ} 29^{\prime} \mathrm{O}$, na região da Serra de Piraputanga que pertence à mesorregião do Pantanal Sul Mato-Grossense ${ }^{19}$.

As bases da economia da região são a pecuária extensiva de corte, o turismo rural e a agricultura de subsistência. A temperatura média anual de $32{ }^{\circ} \mathrm{C}$ é proporcionada pelo clima tropical úmido e sub-úmido, com mínimas de $19^{\circ} \mathrm{C}$ no inverno frio e seco, e máximas de $40{ }^{\circ} \mathrm{C}$ no verão. A precipitação pluviométrica anual está entre 1.000 e $1.400 \mathrm{~mm}$, sendo dezembro e janeiro os meses mais chuvosos. Esta região caracteriza-se por extensas planícies, onde a planície pantaneira representa $70 \%$ da área e $30 \%$ de planalto. Os solos, em sua maioria, são arenosos e pobres, com pequenas manchas argilosas e calcáreas. A vegetação é típica de cerrado, marcada pela presença da Serra de Maracajú, sendo que esses vilarejos se encontram rodeados por morros e estão às margens do Rio Aquidauana. 


\section{Amostragem}

Nove amostras de líquens (5 corticícolas e 4 saxícolas) foram coletadas nos vilarejos de Piraputanga e Palmeiras em 2002. Os líquens foram removidos de seus substratos, armazenados em sacos de papel, codificados e encaminhados ao laboratório. A identificação taxonômica foi feita pela Profa. Dra. M. Fleig (UFRGS) e Prof. Dr. M. P. Marcelli (IB-SP). Cada espécie, após remoção do substrato e limpeza, foi triturada em moinho tipo Willey e o pó resultante foi armazenado em frasco âmbar, mantido em refrigerador.

O líquen Parmotrema tinctorum (Nyl.) Hale, foi amostrado de dois substratos - rocha e córtex de árvore. Pseudoparmelia sphaerospora (Nyl.) Hale, Parmotrema dilatatum (Vain.) Hale, Usnea subcavata Motyka e Pseudoparmelia hypomiltha (Fée) Hale foram coletados de cascas de árvores. Parmotrema miranda (Hale) Hale, Parmotrema flavescens (Kremplh.) Nyl. e Parmotrema delicatulum (Vain.) Hale foram amostrados sobre rocha. Todos os líquens são de morfologia foliosa, exceto a espécie Usnea subcavata Motyka de morfologia fruticosa. A localização geográfica do sítio de amostragem é apresentada na Figura 1.

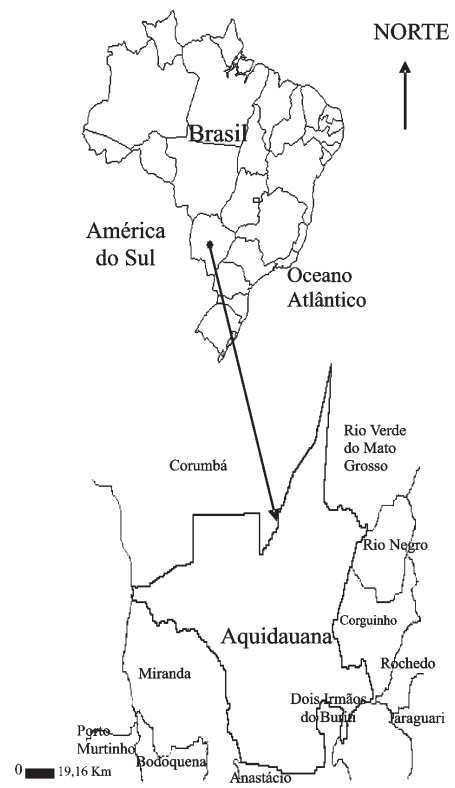

Figura 1. Localização geográfica do sítio de amostragem

\section{Mineralização da amostra}

Efetuou-se um estudo comparativo do tratamento da amostra com ácido nítrico e com a mistura dos ácidos nítrico e perclórico. Para a amostra de líquen $P$. tinctorum foram detectados na mineralização com ácido nítrico $710 \pm 20,2$ e 85,0 $\pm 11,3 \mathrm{mg} \mathrm{kg}^{-1}$ para as espécies $\mathrm{Fe}$ e $\mathrm{Mn}$, respectivamente, enquanto para a mineralização com os ácidos nítrico e perclórico (25:1) foram encontrados $888 \pm 9,25 \mathrm{mg}$ $\mathrm{kg}^{-1}$ para o íon ferro e $96,6 \pm 4,83 \mathrm{mg} \mathrm{kg}^{-1}$ para o íon manganês. Em relação ao $\mathrm{Cu}$ foram detectados $7,75 \pm 2,75 \mathrm{mg} \mathrm{kg}^{-1}$ na mineralização com ácido nítrico e $3,68 \pm 0,872 \mathrm{mg} \mathrm{kg}^{-1}$ na mineralização com os ácidos nítrico e perclórico. O tratamento da amostra com a mistura dos ácidos nítrico e perclórico apresentou melhor repetitividade entre as replicatas e valores de concentração ligeiramente superiores para os íons metálicos $\mathrm{Fe}$ e $\mathrm{Mn}$ e inferior para $\mathrm{Cu}$. No processo de mineralização, dependendo da natureza da amostra, de 2 a $50 \%$ do carbono original permanece. Se a oxidação do carbono for parcial, e dependendo do grau de oxidação, a determinação do elemento sofrerá interferência em menor ou maior grau, dos produtos de decomposição orgânica ${ }^{20}$. Com base nos resultados desse estudo preli- minar, as dissoluções foram efetuadas utilizando-se os ácidos nítrico e perclórico, como descrito a seguir.

Massas de aproximadamente $1,0 \mathrm{~g}$ das amostras (precisão analítica de $\pm 0,01 \mathrm{mg}$ ), previamente secas em estufa a $60^{\circ} \mathrm{C}$ até peso constante, foram pesadas e transferidas para um tubo de politetrafluoretileno (PTFE). Adicionou-se à amostra um volume de $10 \mathrm{~mL}$ de ácido nítrico concentrado (P.A., Merck) e $0,4 \mathrm{~mL}$ de ácido perclórico (P.A., Merck). O tubo fechado foi levado para dentro do cilindro de aço inox (bomba digestora), que foi devidamente fechado. $\mathrm{O}$ sistema foi aquecido em estufa à temperatura de $180{ }^{\circ} \mathrm{C}$ por $3 \mathrm{~h}$. O tubo depois de frio foi removido do sistema digestor e a solução filtrada para um balão volumétrico de $50 \mathrm{~mL}$, cujo volume foi ajustado com água ultrapura obtida por um sistema de purificação Milli-Q.

Após a digestão, o tubo de PTFE foi lavado com detergente Extran MA 02 neutro (Merck) por $30 \mathrm{~min}$ em banho ultra-sônico, enxaguado com água corrente e água destilada. Para minimizar o efeito de memória, o tubo foi imerso em solução de $\mathrm{HNO}_{3}$ a $50 \%$ $(v / v)$ por $12 \mathrm{~h}$ e enxaguado com água deionizada e água ultrapura.

\section{Instrumentação e análise}

As determinações dos íons metálicos nas amostras foram realizadas utilizando-se um espectrômetro de absorção atômica de chama (Varian, modelo 220FS), equipado com lâmpadas de cátodo oco (Techtron), cujas linhas espectrais selecionadas para os elementos foram as seguintes: $\mathrm{Co}=240,7 \mathrm{~nm} ; \mathrm{Cu}=324,7 \mathrm{~nm} ; \mathrm{Cr}=$ $357,9 \mathrm{~nm} ; \mathrm{Fe}=248,3 \mathrm{~nm} ; \mathrm{Mn}=279,5 \mathrm{~nm}$ e $\mathrm{Zn}=231,9 \mathrm{~nm}$.

Para construção das curvas de calibração foram preparadas soluções de padrão misto a partir de soluções-estoque individuais (1000 mg L ${ }^{-1}$ ), adquiridas da Sigma-Aldrich, ou de uma solução intermediária preparada a partir das mesmas. Curvas de calibração com cinco pontos (concentração - Mn: 1,00-3,00 mg L-1; Cr: 0,3001,50 mg L $\mathrm{m}^{-1}$; Co: 0,200-1,00 mg L-1; Cu: 0,0300-0,150 mg L-1; Fe: 2,00-10,0 mg L ${ }^{-1}$; Zn: 0,250-1,25 mg L-1) foram obtidas. As concentrações dos íons metálicos no líquen $P$. tinctorum coletado sobre rocha foram determinadas utilizando-se as curvas de calibração (absorbância vs.concentração) e os valores comparados com aqueles obtidos pelo método de adição de padrão. A amostra foi diluída para que as absorbâncias de Fe e Mn ficassem dentro do intervalo de concentração da curva de calibração. As concentrações obtidas para os íons metálicos $\mathrm{Mn}, \mathrm{Cr}, \mathrm{Zn}$, Co e Fe foram similares nos dois métodos, assim como os valores de inclinação das retas. A quantificação foi efetuada pelo método de rotina, exceto $\mathrm{Cu}$ que foi quantificado pelo método da adição de analito devido à baixa concentração nas amostras. Para análise de $\mathrm{Cu}$, tomaram três alíquotas de 5,0 mL da solução da amostra e volumes crescentes de solução padrão foram adicionados para se obter concentrações de 0,03; 0,06 e 0,09 $\mathrm{mg} \mathrm{L}^{-1}$ em balão volumétrico de 25,0 mL. As curvas de calibração apresentaram valores de $r^{2} \geq 0,99549$. Realizaram-se três determinações para cada espécie de líquen. $\mathrm{O}$ branco de reagente foi preparado pela adição dos ácidos ao tubo de PTFE e submetido ao mesmo tratamento das amostras.

\section{Limite de detecção e recuperação do método}

A sensibilidade do método foi expressa através dos limites de detecção (LOD) e quantificação (LOQ), definidos como 3 e 10 Sd $\mathrm{m}^{-1}$, respectivamente, onde Sd é uma estimativa do desvio padrão das medidas de absorbâncias de 10 brancos e m o coeficiente angular da curva de calibração. Os limites, de acordo com a IUPAC ${ }^{21}$, devem ser calculados utilizando-se as respostas do branco de amostra, amostra de composição igual à analisada, mas sem o analito. 
Neste estudo, seria uma amostra homogênea de líquen sem as espécies metálicas. Devido às dificuldades na obtenção deste, utilizou-se o branco de reagentes, o que subestima o limite de detecção, já que a influência da matriz não é medida ${ }^{22}$.

A eficiência da extração foi determinada no estudo de recuperação pela adição simultânea de padrões ao líquen $P$. dilatatum. Adicionou-se à amostra uma alíquota $(1,0 \mathrm{~mL})$ de solução padrãomisto dos metais, sendo que as concentrações finais na amostra dos padrões adicionados foram: $\mathrm{Cu}=5,00 \mathrm{mg} \mathrm{kg}^{-1} ; \mathrm{Zn}=23,0 \mathrm{mg}$ $\mathrm{kg}^{-1} ; \mathrm{Mn}=200 \mathrm{mg} \mathrm{kg}^{-1} ; \mathrm{Co}=18,0 \mathrm{mg} \mathrm{kg}^{-1} ; \mathrm{Cr}=20,0 \mathrm{mg} \mathrm{kg}^{-1} ; \mathrm{Fe}=$ $300 \mathrm{mg} \mathrm{kg}^{-1}$.

\section{Análise estatística}

A aplicação de métodos de análise estatística multivariada, como ferramenta auxiliar, está cada vez mais sendo recomendada quando se quer alcançar melhores resultados ambientais e deduzir fontes características de contaminação ${ }^{23-26}$.

Neste sentido, para investigar as possíveis correlações entre elementos e líquens foi aplicada análise multivariada ao conjunto de dados, incluindo análise de Pearson e análise de cluster (CA). Todos os cálculos estatísticos foram efetuados utilizando-se o software Minitab ${ }^{27}$ para Windows versão 14.

\section{Pearson}

É um teste paramétrico de correlação linear, no qual os valores das variáveis X e Y são mensurados em nível intervalar ou de razões. $\mathrm{O}$ coeficiente de Pearson -r- pode variar de $-1 \mathrm{a}+1$ e quanto mais próximos desses valores, mais forte a associação das variáveis em estudo. $\mathrm{O}$ escore zero desse coeficiente indica ausência de correlação. A probabilidade de significância do teste (p-valor) corresponde ao menor nível de significância que pode ser assumido para rejeitar a hipótese nula. Diz-se que há significância estatística quando pvalor é menor que o nível de significância adotado. Normalmente o nível de significância adotado para o p-valor é $\mathrm{p} \leq 0,05$.

\section{Análise de cluster}

Com procedimentos multivariados de cluster, objetos ou indivíduos, descritos por um conjunto de medidas, podem ser classificados com base na semelhança ou dessemelhança de suas características. Similaridade e dissimilaridade dos indivíduos são descritas pelas distâncias euclidianas dos valores das características do indivíduo. O objetivo da análise de cluster é aperfeiçoar a heterogeneidade das classes (grupos, clusters) assim como a homogeneidade dentro deles ${ }^{28}$. A partir da análise de cluster, pode-se identificar indivíduos com características semelhantes e, então, criar grupos com estes indivíduos. Diferentes algoritmos de cluster cumprem este objetivo. Neste estudo foi utilizado o método aglomerativo de Ward, o qual maximiza a variância entre os grupos e minimiza-a entre os membros do mesmo grupo ${ }^{29}$. A medida de distância utilizada foi a distância do coeficiente de correlação. Para mostrar os resultados em cluster, dendrogramas são usados como forma de apresentação visual dos passos da técnica hierárquica de cluster.

\section{RESULTADOS E DISCUSSÃO}

Os limites de detecção para os íons de $\mathrm{Co}, \mathrm{Mn}, \mathrm{Fe}, \mathrm{Cr}, \mathrm{Zn}$, e $\mathrm{Cu}$ foram de 0,$047 ; 0,016 ; 0,049 ; 0,062 ; 0,045$ e $0,010 \mathrm{mg} \mathrm{L}^{-1}$, enquanto que os limites de quantificação para os mesmos elementos foram 0,$156 ; 0,054 ; 0,164 ; 0,206 ; 0,150 ; 0,033 \mathrm{mg} \mathrm{L}^{-1}$, respectivamente. Os valores de recuperação para $\mathrm{Cr}, \mathrm{Mn}, \mathrm{Co}, \mathrm{Zn}$ e $\mathrm{Cu}$ foram de $95,6 \pm 3,91 \% ; 99,4 \pm 4,90 \% ; 110 \pm 4,17 \% ; 82,7 \pm 2,14 \% ; 97,9$ $\pm 3,30 \%$, respectivamente.

Esta é a primeira publicação científica a relatar concentrações de íons metálicos em líquens do cerrado sul-mato-grossense. Os valores médios de concentrações para os íons metálicos nas 9 espécies de líquens são apresentados na Tabela 1. Dentre os íons inorgânicos estudados, Fe e Mn foram os mais abundantes em todas as amostras, enquanto o íon $\mathrm{Cu}$ apresentou a menor concentração, Figuras 2 e 3 . Para os íons Fe a maior concentração foi de $1,57 \times 10^{3} \mathrm{mg} \mathrm{kg}^{-1}$ no líquen $P$. Miranda e a menor concentração foi de $248 \mathrm{mg} \mathrm{kg}^{-1}$ no líquen $U$. Subcavata. O maior valor de concentração para a espécie inorgânica Mn foi encontrado na amostra $P$. hypomiltha $(397 \mathrm{mg} \mathrm{kg}$ $\left.{ }^{1}\right)$ e o menor valor na amostra $P$. tinctorum coletado em rocha $(98,5$ $\mathrm{mg} \mathrm{kg}^{-1}$ ). A espécie $U$. subcavata, coletada de casca de árvore, que apresenta uma morfologia fruticosa, não só apresentou baixa concentração para o íon $\mathrm{Cu}$, mas também para $\mathrm{Cr}$, Zn e Fe em relação às

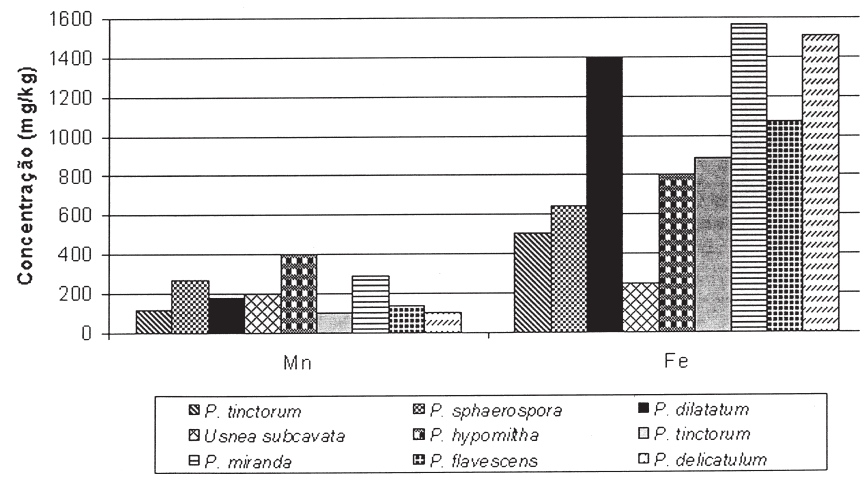

Figura 2. Concentração de Mn e Fe nas nove espécies de líquens

Tabela 1. Valores médios de concentração dos íons metálicos nas nove espécies de líquens

\begin{tabular}{|c|c|c|c|c|c|c|c|}
\hline \multirow[t]{2}{*}{ Líquens } & \multicolumn{7}{|c|}{ Concentração em mg kg-1 } \\
\hline & $\mathrm{S}^{*}$ & $\mathrm{Cr}$ & $\mathrm{Mn}$ & $\mathrm{Co}$ & $\mathrm{Zn}$ & $\mathrm{Fe}$ & $\mathrm{Cu}$ \\
\hline Parmotrema tinctorum & $\mathrm{c}$ & 18,54 & 118,33 & 10,08 & 22,08 & 507,41 & 4,94 \\
\hline Pseudoparmelia sphaerospora & $\mathrm{c}$ & 44,26 & 268,92 & 13,54 & 34,79 & 643,20 & 6,52 \\
\hline Parmotrema dilatatum & $\mathrm{c}$ & 25,97 & 179,86 & 13,04 & 18,08 & 1398,06 & 5,94 \\
\hline Usnea subcavata & $\mathrm{c}$ & 18,24 & 196,67 & 20,36 & 14,62 & 248,41 & 3,23 \\
\hline Pseudoparmelia hypomiltha & $\mathrm{c}$ & 18,80 & 397,33 & 21,85 & 27,63 & 806,23 & 4,46 \\
\hline Parmotrema tinctorum & $\mathrm{r}$ & 37,73 & 98,50 & 22,34 & 20,37 & 888,45 & 3,68 \\
\hline Parmotrema miranda & $\mathrm{r}$ & 26,21 & 286,49 & 17,88 & 31,20 & 1568,01 & 5,60 \\
\hline Parmotrema flavescens & $\mathrm{r}$ & 22,22 & 135,64 & 24,81 & 19,24 & 1071,61 & 7,57 \\
\hline Parmotrema delicatulum & $\mathrm{r}$ & 21,23 & 101,37 & 16,98 & 20,82 & 1514,52 & 7,51 \\
\hline
\end{tabular}

$\mathrm{S}^{*}=$ substrato; $\mathrm{c}=$ caule; $\mathrm{r}=$ rocha; 


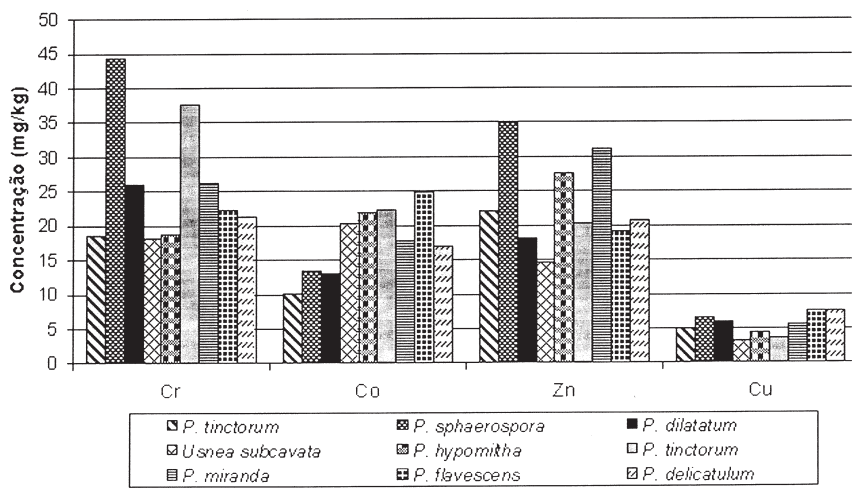

Figura 3. Concentração de $\mathrm{Cr}$, Co, Zn e Cu nas nove espécies de líquens

outras amostras de líquens analisadas. Líquens do gênero Parmotrema, desenvolvidos sobre rocha (P. tinctorum, P. delicatulum, $P$. flavescens e P. miranda) apresentaram concentrações de Fe superiores às do líquen de mesma espécie, $P$. tinctorum, e as do gênero Pseudoparmelia (P. sphaerospora e P. hypomiltha), ambos desenvolvidos sobre casca de árvores.

Os líquens são considerados organismos acumuladores de elementos químicos do ambiente por serem sensíveis à poluição e pouco sensíveis aos efeitos tóxicos dos elementos ${ }^{30}$. Valores elevados de concentração de íons metálicos nos líquens têm sido atribuídos à poluição do ar, no entanto, solos contaminados e/ou a composição mineral do solo pode influenciar ${ }^{31}$. Os processos naturais, como a ressuspensão de partículas do solo pelos ventos, salsugem e emanações vulcânicas podem também proporcionar o enriquecimento de íons metálicos nos líquens. Espécies inorgânicas, como $\mathrm{Al}, \mathrm{Si}, \mathrm{Ca}, \mathrm{Fe}$, Ti e Mn, são características da ressuspensão do $\mathrm{solo}^{30}$. O intemperismo é um fenômeno que pode disponibilizar para o ambiente metais contidos em rochas ${ }^{32}$.

A criação extensiva de gado bovino e a agricultura são atividades desenvolvidas no local de amostragem dos líquens, assim como em outras regiões do estado de Mato Grosso do Sul, representando as bases da economia. Estas atividades estão intimamente ligadas ao uso intensivo de agroquímicos e outras técnicas de manejo que podem poluir o meio ambiente. Íons metálicos, como $\mathrm{Fe}, \mathrm{Mn}, \mathrm{Co}$, $\mathrm{Zn}$ e $\mathrm{Cu}$, são normalmente encontrados nos suplementos minerais para bovinos. $\mathrm{O}$ íon cromo vem sendo utilizado em dietas de ruminantes ${ }^{33,34}$, principalmente em situações de estresse (doenças, gestação e transporte), sendo também usado no curtimento mineral de couro $^{35-37}$. Segundo dados do IBGE no estado de Mato Grosso do Sul, no terceiro trimestre de 2006, foram curtidos ao cromo 1.068.654 couros, $11,22 \%$ da produção nacional ${ }^{38}$. O uso de agroquímicos, de suplementos minerais e o aproveitamento de resíduos agroindustriais (lodo de curtume) para adubação podem causar impactos ambientais, afetando o ecossistema com a contaminação de solos, cursos d'água, atmosfera e alimentos. Sabe-se que as espécies herbáceas e muitas espécies arbóreas que crescem em solo contaminado por metais pesados apresentam teores foliares elevados de metais, em muitos casos, superiores aos níveis críticos de toxidez ${ }^{39}$. Provavelmente, a combustão de biomassa, que possui altos teores de metais, pode contribuir para elevar sua concentração na atmosfera por emissão direta de material particulado ou ressuspensão das cinzas, propiciando o enriquecimento de espécies químicas nos líquens. Em áreas de Cerrado esta prática é muito comum, com propósito de promover a renovação ou recuperação das pastagens. A queimada que deveria ser pequena e controlada acaba muitas vezes se espalhando, devido à baixa precipitação pluviométrica no Estado de junho a setembro. Sabe-se que a combustão de biomassa é uma importante fonte de emissão de partículas finas (diâmetro aerodinâmico inferior a $10 \mu \mathrm{m}$ ), e estas têm um tempo longo de residência na atmosfera (entre 100 e 1000 h) podendo ser transportadas por longas distâncias $(100 \text { a } 1000 \mathrm{~km})^{40}$.

Os íons Cr, Co e Mn apresentaram concentrações nos líquens acima do valor de referência. A Tabela 2 apresenta valores médios comparativos entre as concentrações obtidas neste estudo com os realizados na Itália, Brasil e Estados Unidos. Bergamaschi et al. ${ }^{31}$ efetuaram a determinação de 23 elementos nos líquens coletados em áreas de grande elevação, consideradas não poluídas na Itália, no Nepal e Quênia. A análise elementar dos líquens nestes locais forneceu valores da composição natural dos líquens que podem ser tomadas como referência para amostras de áreas não poluídas. Coccaro et al. $^{41}$ estudaram as concentrações de 30 elementos em líquens amostrados no Jardim Botânico de São Paulo (reserva biológica de Mata Atlântica), enquanto Williams et al. ${ }^{42}$ relataram a concentração média de metais em líquens coletados em 5 áreas de deserto no sudoeste americano. As concentrações médias dos íons $\mathrm{Cr}, \mathrm{Mn}$ e Co nos líquens estudados foram superiores aos valores de referência de áreas não poluídas da Itália, Nepal e Quênia ${ }^{31}$ (Tabela 2). Também são superiores aos valores obtidos para líquens do Jardim Botânico de São Paulo $^{40}$ e do deserto americano ${ }^{42}$ (área não impactada), sugerindo uma provável contribuição de fontes antropogênicas no acúmulo destes elementos nos líquens.

Com o intuito de diagnosticar as prováveis fontes de contaminação, o teste de correlação linear de Pearson e a análise de cluster foram aplicados às concentrações para verificar as correlações e similaridade entre os íons metálicos. A análise de correlação de Pearson entre concentrações normalizadas dos íons metálicos nos líquens mostrou fracas correlações entre os elementos, exceto entre $\mathrm{Mn}$ e $\mathrm{Zn}(\mathrm{r}=0,628, \mathrm{p}=0,070)$ e entre Co e Fe $(\mathrm{r}=0,594, \mathrm{p}=$ 0,092), Tabela 3. O dendrograma dos elementos revelou quatro clusters: (1) Cr; (2) Mn e Zn; (3) Co; (4) Fe e Cu (Figura 4). Níveis de similaridade de 81,4 e de 79,6\% foram obtidos entre Mn e Zn e entre $\mathrm{Fe}$ e $\mathrm{Cu}$, respectivamente, complementando o teste de correlação linear de Pearson.

Os coeficientes de correlação de Pearson mostraram associações sem significância entre as concentrações dos íons $\mathrm{Cr}$, Mn e

Tabela 2. Valores médios de concentração $\left(\mathrm{mg} \mathrm{kg}^{-1}\right)$ para os íons metálicos em diferentes estudos

\begin{tabular}{|c|c|c|c|c|c|c|}
\hline \multirow[t]{2}{*}{ Íons Metálicos } & \multicolumn{2}{|c|}{ Neste estudo } & \multicolumn{2}{|c|}{ Itália $^{31}$} & \multirow{2}{*}{$\begin{array}{l}\text { São Paulo }{ }^{41} \\
\text { Média * } \pm S\end{array}$} & \multirow{2}{*}{$\begin{array}{c}\text { EUA }^{42} \\
\text { Média** }\end{array}$} \\
\hline & Média $* \pm S$ & $\mathrm{CV}(\%)$ & Média $* \pm S$ & $\mathrm{CV}(\%)$ & & \\
\hline $\mathrm{Cr}$ & $25,91 \pm 9,19$ & 35,45 & $1,17 \pm 1,3$ & 79,4 & $3,6 \pm 1,3$ & 6,6 \\
\hline $\mathrm{Mn}$ & $198,12 \pm 101,49$ & 51,23 & $108 \pm 75$ & 69,7 & $17 \pm 8$ & 89 \\
\hline Co & $17,86 \pm 4,92$ & 27,55 & $0,25 \pm 0,12$ & 44,9 & $\mathrm{x}$ & 7,0 \\
\hline $\mathrm{Zn}$ & $23,20 \pm 6,59$ & 28,44 & $35 \pm 16$ & 44,4 & $62 \pm 20$ & 7,5 \\
\hline $\mathrm{Fe}$ & $960,66 \pm 463,93$ & 48,29 & $220 \pm 105$ & 47,9 & $764 \pm 333$ & 1700 \\
\hline $\mathrm{Cu}$ & $5,49 \pm 1,56$ & 28,41 & $4,1 \pm 3,1$ & 76,9 & $\mathrm{x}$ & 23 \\
\hline
\end{tabular}

$\mathrm{S}=$ desvio padrão; $\mathrm{CV}=$ coeficiente de variância; $\mathrm{x}=$ valor não detectado; $*$ = média aritmética; $* *$ média geométrica 
Tabela 3. Valores dos coeficientes de correlação de Pearson (r) entre os íons metálicos e os p-valores

\begin{tabular}{|c|c|c|c|c|c|}
\hline & $\mathrm{Cr}$ & $\mathrm{Mn}$ & Co & $\mathrm{Zn}$ & $\mathrm{Fe}$ \\
\hline $\mathrm{Mn}$ & $0,002(0,996)$ & & & & \\
\hline $\mathrm{Co}$ & $-0,106(0,786)$ & $0,100(0,797)$ & & & \\
\hline $\mathrm{Zn}$ & $0,514(0,156)$ & $0,628(0,070)$ & $-0,225(0,561)$ & & \\
\hline $\mathrm{Fe}$ & $0,013(0,974)$ & $-0,063(0,872)$ & $0,020(0,959)$ & $0,136(0,728)$ & \\
\hline $\mathrm{Cu}$ & $0,080(0,838)$ & $-0,181(0,642)$ & $-0,123(0,753)$ & $0,219(0,571)$ & $0,594(0,092)$ \\
\hline
\end{tabular}

Obs: entre parênteses p-valor

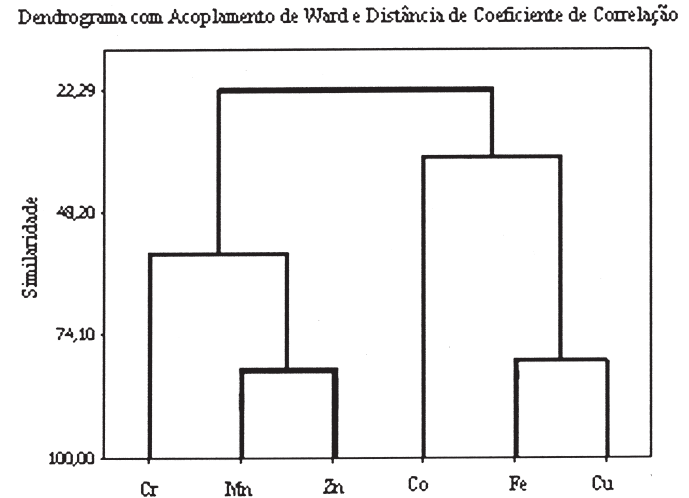

Figura 4. Dendrograma obtidos para os íons metálicos aplicando-se a análise de cluster

Co nos líquens ( $\mathrm{r} \leq 0,100$ e $\mathrm{p} \geq 0,797$ ); o dendrograma apresentou clusters distintos para estes elementos, evidenciando diferentes fontes principais de contaminação.

Como discutido anteriormente, o processo de curtimento do couro ao cromo pode ser a fonte de contaminação deste elemento. Quanto ao íon Mn, a exploração da jazida mineral do Maciço de Urucum pode ser uma fonte de contaminação adicional as já anteriormente citadas. O Maciço está localizado nos municípios de Corumbá e Ladário e vem sendo explorado desde 1907. Em Corumbá encontra-se também uma siderurgia que utiliza o Mn para produzir ligas metálicas. Estas certamente são fontes de emissão de poluentes do ar e, conseqüentemente, de deposição atmosférica dos mesmos. Neste contexto, os mecanismos de transporte de poluentes atmosféricos de longas distâncias podem estar eventualmente envolvidos. O Co é muito utilizado no cultivo de grãos, principalmente da soja, para fixação biológica de nitrogênio. Este é utilizado no tratamento das sementes ou aplicado na parte aérea das plantas. Em solos com deficiência de cobalto, a aplicação do nutriente é de até $2 \mathrm{~kg} /$ ha de sulfato de cobalto ( $21 \%$ de Co).

Para caracterizar a capacidade dos três diferentes gêneros de líquens em acumular íons metálicos, testes de análise multivariada de Pearson e de cluster também foram aplicados. O teste de correlação de Pearson foi executado utilizando-se os valores normalizados das concentrações dos íons metálicos. Foram observadas fortes correlações positivas para as concentrações dos íons metálicos entre os líquens dos gêneros Parmotrema e Pseudoparmelia, e entre Usnea e Pseudoparmelia $(0,911 \leq \mathrm{r} \leq 1,000$ e $0,000 \leq \mathrm{p} \leq 0,012)$, Tabela 4. O líquen do gênero Usnea (subcavata) mostrou os menores valores de correlação $(0,777 \leq \mathrm{r} \leq 0,866$ e $0,026 \leq \mathrm{p} \leq 0,069)$ com o gênero Parmotrema (tinctorum, miranda, flavescens e dilatatum, delicatulum).

Aplicando a análise de cluster obteve-se o dendrograma para os líquens, mostrado na Figura 5. Dois principais clusters são formados (1 e 2), sendo que cada cluster subdivide-se em dois subclusters: 1a ( $P$. tinctorum $r$ e $P$. mirada), 1 b ( $P$. dilatatum, $P$. flavescens, $P$. tinctorum $c, P$. dilecatulum), 2a ( $P$. sphaerospora, $P$. hypomiltha) e $2 \mathrm{~b}$ (usnea subcavata). O cluster 2 revelou elevada similaridade entre os líquens do gênero Pseudoparmelia $(99,7 \%)$ e este com o gênero Usnea sp. (97,0\%), assim como o cluster 1 mostrou similaridade superior a 99,3\% entre as espécies do gênero Parmotrema.

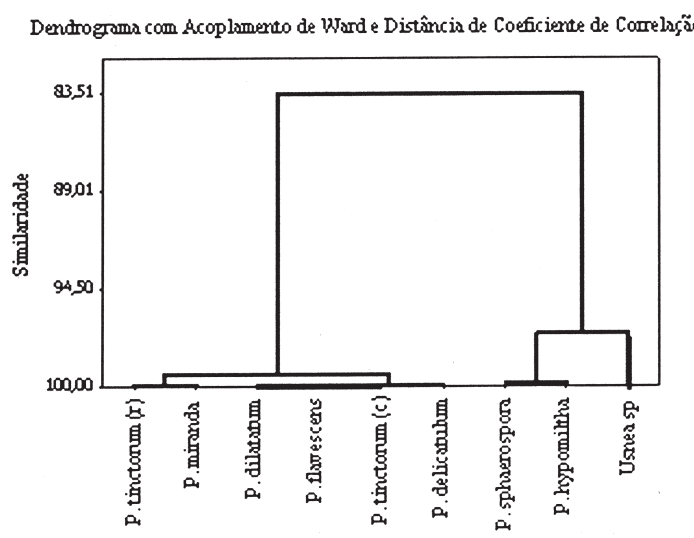

Figura 5. Dendrograma obtido para os líquens aplicando a análise de cluster

\section{CONCLUSÃO}

A aplicação de líquens como biomonitores ambientais parece ser uma boa maneira de se monitorar a qualidade da poluição at-

Tabela 4. Valores dos coeficientes de correlação de Pearson (r) entre os líquens e os p-valores

\begin{tabular}{|c|c|c|c|c|c|c|c|c|}
\hline & P. tinctorum & P. sphaerospora & P. dilatatum & Usnea subcavata & a P. hypomiltha & P. $\operatorname{tinctorum}(r)$ & P. miranda & P. flavescens \\
\hline P. sphaerospora & $0,983(0,000)$ & & & & & & & \\
\hline P. dilatatum $(c)$ & $0,995(0,000)$ & $0,960(0,002)$ & & & & & & \\
\hline Usnea subcavata & $0,866(0,026)$ & $0,942(0,005)$ & $0,815(0,048)$ & & & & & \\
\hline P. hypomiltha & $0,964(0,002)$ & $0,996(0,000)$ & $0,935(0,006)$ & $0,967(0,002)$ & & & & \\
\hline P. tinctorum $(r)$ & $0,992(0,000)$ & $0,952(0,003)$ & $0,999(0,000)$ & $0,798(0,057)$ & $0,924(0,008)$ & & & \\
\hline P. miranda & $0,999(0,000)$ & $0,974(0,001)$ & $0,999(0,000)$ & $0,845(0,034)$ & $0,953(0,003)$ & $0,996(0,000)$ & & \\
\hline P. flavescens & $0,995(0,000)$ & $0,958(0,003)$ & $1,000(0,000)$ & $0,811(0,050)$ & $0,932(0,007)$ & $1,000(0,000)$ & $0,998(0,000)$ & \\
\hline P. delicatulum & $0,987(0,000)$ & $0,941(0,005)$ & $0,998(0,000)$ & $0,777(0,069)$ & $0,911(0,012)$ & $0,999(0,000)$ & $0,993(0,000)$ & $0,999(0,000)$ \\
\hline
\end{tabular}

Obs: entre parênteses p-valor 
mosférica. As concentrações para os seis íons metálicos avaliados nas 9 espécies de líquens compreenderam valores desde 3,23 mg $\mathrm{kg}^{-1}(\mathrm{Cu})$ até $1,57 \times 10^{3} \mathrm{mg} \mathrm{kg}^{-1}(\mathrm{Fe})$. Apesar dos altos valores encontrados para o íon $\mathrm{Fe}$, este ainda se encontra abaixo dos níveis considerados de referência. A composição mineral do solo pode ser em parte responsável pelas elevadas concentrações deste íon nos líquens, devido a processos naturais como a ressuspensão de partículas do solo pelos ventos.

Quanto ao Co, Mn e Cr, estes sim estão acima dos valores obtidos em áreas não poluídas, considerados de referência.

Os resultados obtidos pela aplicação do coeficiente de correlação linear de Pearson e da análise de cluster permitiram inferir prováveis fontes de contaminação para os íons metálicos. A análise de Pearson mostrou fracas correlações $(r \leq 0,100$ e $p \geq 0,786$ ) entre os íons $\mathrm{Co}, \mathrm{Mn}$ e $\mathrm{Cr}$, e a de cluster apresentou esses íons em clusters distintos, evidenciando fontes antropogências diferentes de contaminação. Atividades ligadas à agricultura, agroindústria e exploração de jazidas minerais podem estar contribuindo para o enriquecimento dos íons $\mathrm{Cr}, \mathrm{Mn}$, Co nos líquens.

Fortes correlações positivas de Pearson foram obtidas entre os líquens dos gêneros Parmotrema e Pseudoparmelia, e entre Usnea e Pseudoparmelia $(\mathrm{r} \geq 0,911$ e $\mathrm{p} \leq 0,012)$.

A análise de cluster revelou quatro subclusters: 1a e 1b formados pelos líquens do gênero Parmotrema, enquanto os grupos $2 \mathrm{a}$ e $2 \mathrm{~b}$ foram representados pelos líquens dos gêneros Pseudoparmelia e Usnea, respectivamente. Isto é uma evidência que os três gêneros de líquens se diferenciam quanto à capacidade de acumular os íons metálicos, destacando-se o gênero Usnea sp. com menor capacidade, por possuir morfologia fruticosa.

\section{AGRADECIMENTOS}

Ao $\mathrm{CNPq} /$ Fundect pela bolsa de Iniciação Científica; ao Departamento de Química da UFMS pelos equipamentos cedidos, à Profa. Dra. M. Fleig e ao Prof. Dr. M. P. Marcelli pela identificação dos líquens, e ao Prof. Dr. L. Sauer pela colaboração na interpretação dos resultados estatísticos.

\section{REFERÊNCIAS}

1. Marcelli, M. P. Em Biodiversidade do estado de São Paulo, Brasil 2 Fungos Macroscópicos \& Plantas; Bicudo, C. E. M.; Shepherd, G. J., eds.; Ed. São Paulo: São Paulo, 1998, p. 27.

2. Kappen, L. Em The Lichens; Ahmadjian, V.; Hale, M. E., eds.; Academic Press: New York, 1973, p. 311.

3. Brodo, I. M. Em ref. 2, p. 401.

4. Hale Jr., M. E. Em The Biology of Lichens; $3^{\text {rd }}$ ed.; Edward Arnold: Baltimore, 1983.

5. Puckett, K. J.; Bibliotheca Lichenologica 1988, 30, 231.

6. Rossbach, M.; Jayasekera, R.; Kniewald, G.; Thang, M. H.; Sci. Total Environ. 1999, 232, 59
7. Wappelhorst, O.; Kuhn, I.; Oehlmann, J.; Markert, B.; Sci. Total Environ. 2000, 249, 243.

8. Carreras, H. A.; Pignata, M. L.; Environ. Pollut. 2001, 111, 45.

9. Sawidis, T.; Chettri, M. K.; Zachariadis, G. A.; Stratis, J. A.; Ecotoxicol. Environ. Saf. 1995, 32, 73

10. Monaci, F.; Bargagli, R.; Gasparo, D.; Acta Bot. Neerl. 1997, 46, 403.

11. Scerbo, R.; Possenti, L.; Lampugnani, L.; Ristori, T.; Barale, R.; Barghigiani, C.; Sci. Total Environ. 1999, 241, 91.

12. Loppi, S.; Ivanov, D.; Boccardi, R.; Environ. Pollut. 2002, 116, 123.

13. Branquinho, C.; Brown, D. H.; Maguas, C.; Catarino, F.; Environ. Exp. Bot. 1997a, 37, 95 .

14. Branquinho, C.; Brown, D. H.; Maguas, C.; Catarino, F.; Environ. Exp. Bot. 1997b, 38,165

15. Chettri, M. K.; Cook, C. M.; Vardaka, E.; Sawidis, T.; Lanaras, T.; Environ. Exp. Bot. 1998, 39, 1.

16. Kauppi, M.; Kauppi, A.; Garty, J.; New Phytol. 1998, 139, 537.

17. Garty, J.; Crit. Rev. Plant Sci. 2001, 20, 309.

18. Glenn, M. G.; Gomez-Bolea, A.; Lobello, R.; Lichenol. 1995, 27, 291.

19. IBGE/SEPLAN - Instituto de Geografia e Estatística do Brasil. Secretaria de Planejamento e Coordenação do Governo do Mato Grosso do Sul; Atlas Multirreferencial, Aquidauana: IBGE, 2000.

20. Krug, F. J.; I Workshop on Methods of Sample Decomposition - PréTratamento de Amostras, Centro de Energia Nuclear na Agricultura de Piracicaba, Brasil, 1996.

21. Analytical Methods Commitee, Analyst 1987, 112, 199

22. Limaverde-Filho, A. M.; Campos, R. C.; Quim. Nova 1999, 22, 477.

23. Carreras, H. A.; Wannaz, E. D.; Perez, C. A.; Pignata, M. L.; Environ. Res. 2005, 97, 50 .

24. Siu-lan Lee, C.; Li, X.; Shi, W.; Ching-nga Cheug, S.; Thornton, I.; Sci. Total Environ. 2006, 356, 45.

25. Loppi, S.; Pirintsos, S. A.; Environ. Pollut. 2003, 121, 327.

26. Yenisoy-Karakas, S.; Tuncel, S. G.; Sci. Total Environ. 2004, 329, 43.

27. Ryan Jr, T. A.; Ryan, B. F.; Joiner, B. L.; MINITAB - Statistical Software, Universidade do Estado da Pensilvânia, EUA, 1972.

28. Pesch, R.; Schroeder, W.; Ecological Indicators, no prelo.

29. Gallego, J. L. R.; Ordónez, A.; Loredo, J.; Environ. Int. 2002, 27, 589.

30. Helena, P. N.; Franc, B.; Cvetka, R. L.; J. Atmos. Chem. 2004, 49, 223

31. Bergamaschi, L.; Rizzio, E.; Giaveri, G.; Profumo, A.; Loppi, S.; Gallorini, M.; Chemosphere 2004, 55, 933.

32. Soares, C. R. F. S.; Accioly, A. M. A.; Marques, T. C. L. L. S. M.; Siqueira, J. W.; Moreira, F. M. S.; Rev. Bras. Fisiol. Veg. 2001, 13, 302.

33. Kegley, E. B.; Spears, J. W.; Eisemann, J. H.; J. Dairy Sci. 1997, 80, 1744.

34. Hayirli, A.; Bremmer, D. R.; Bertics S. J.; Socha, M. T.; Grummer, R. R.; J. Dairy Sci. 2001, 84, 1218.

35. Berry, F. J.; Constantini, N.; Smart, L. E.; Waste Management 2002, 22, 761.

36. Mignini, F.; Streccioni, V.; Baldo, M.; Vitali, M.; Indraccolo, U.; Bernacchia, G.; Cocchioni, M.; Preventive Medicine 2004, 39, 767.

37. Tariq, S. R.; Shah, M. H.; Shaheen, N.; Khalique, A.; Manzoor, S.; Jaffar, M.; J. Hazard. Mater. 2005, 122, 17

38. http://www.sidra.ibge.gov.br/bda/ acessada em Dezembro 2006.

39. Gisbert, C.; Clemente, R.; Navarro-Avi, J.; Baixauli, C.; Ginér, A.; Serrano, R.; Walker, D. J.; Bernal, M. P.; Environ. Exp. Bot. 2006, 56, 19.

40. Lara, L. L.; Artaxo, P.; Martinelli, L. A.; Camargo, P. B.; Victoria, R. L.; Ferraz, E. S. B.; . Atmos. Environ. 2005, 39, 4627

41. Coccaro, D. M. B.; Saiki, M.; Vasconcellos, M. B. A.; Marcelli, M. P.; J. Radioanal. Nucl. Chem. 2000, 244, 141.

42. Williamns, R. N.; Casellas, R. C.; Mangelson, N. F.; Rees, L. B.; St. Clair, L. L.; Schaalje, G. B.; Swalderg, K. D.; Nucl. Instrum. Methods Phys. Res., Sect. B 1996, 109/110, 336. 